\section{Movements of Lugworms}

A RECENT review of the hydrostatic skeleton in invertebrates by Chapman ${ }^{1}$ includes the following sentence about Arenicola: "Wells (1954) has shown that the three anterior septa are connected with the retention of the proboscis against the normal resting or tonus pressure maintained by the bodywall in the colom, which would otherwise be sufficient to cause protrusion of the proboscis". This is untrue ; I did not show, or claim to show, what he ascribes to me. The paper to which he refers ${ }^{2}$ contains an analysis of the working of the very intricate proboscis mechanism. The first of the three anterior septa is differentiated into several parts and actively assists both the extrusion and the withdrawal of the proboscis, the details varying somewhat with the species; the extent of its responsibility for keeping the resting proboscis in position is unknown. Neither of the other two anterior septa has anything to do with the movements or position of the proboscis.

In the same review Chapman writes that in errant polychætes "the oblique muscles run from a lateral position to a point below the nerve cord" but in Arenicola "no oblique muscles are present", that "the lugworm cannot crawl", and that eversion of the proboscis is assisted by "a simple contraction of the posterior end of the lugworm". In fact, of course, the oblique muscles of Arenicola are numerous and conspicuous in dissections ${ }^{3}$, the worms crawl actively in their burrows, in glass tubes or on the surface of the sand ${ }^{4}$, and, as anyone who has watched a vigorously burrowing lugworm will agree, the description of the movements of its hinder part as simple is wide of the mark. He appears to regard the parapodial musculature as largely or entirely derived from the circular muscle layer of the body wall ; in fact, as one would expect on functional grounds, the chief parapodial muscles run longitudinally and are derived from the longitudinal layer ${ }^{5}$. It is regrettable that such inexact statements have been put into circulation when accurate information about the movements of lugworms, and the structures which bring them about, was already available.

Department of Zoology, University College,

London, W.C.1. Aug. 1.

1 Chapman, G., Biol. Rev., 33, 338 (1958).

2 Wells, G. P Quart. J. Micr. Sci., 95, 251 (1954).

${ }^{3}$ Ashworth, J. H., Liverpool Marine Biology Committee Memoirs, xi (London, 1904). Wells, G. P., J. Mar. Biol. Assoc., 29, 1 (1950).

- Guberlet, J. E., Proc. Fifth Pacific Sci. Congr., 5, 4213 (1934). Just, B., $Z$. vergl.' Physiol., 2, 155 (1924) MacGinitie, G., E., and MacGinitie, N., "Natural History of Marine Animals" (New York, 1949). Wells, G. P., Symp. soc. Exp. Biol., No. 4, 127 (1950) Werner, B., Helgol. wiss. Meeresunters., 5, 343 (1956).

'Lillie, R. S., Mitt. zool. Sta. Neapel, 17, 341 (1905). Wells, G. P. Proc. Zool. Soc. Lond., 114, 100 (1944).

\section{Striations of Skeletal Muscle}

IT seems to have been generally assumed that the structure of myofibrils in skeletal, or striated, muscle is fundamentally similar in all the vertebrate classes, and, in addition, observations on invertebrates, particularly insects, have been widely applied to the muscles of vertebrates. In fact, much of the detailed morphological description of striations of mammalian muscle in histological text-books appears to be based on work on insects, as can be seen by reference to Schafer's well-known text-book ${ }^{1}$, or to Cowdry's work on special cytology ${ }^{2}$.
This assumption of structural uniformity in all animals is supported by the appearance as seen by using crossed 'Polaroid' glasses, or crossed Nicol prisms, and by staining with Heidenhain's iron hæmatoxylin method.

However, use of a wider range of staining methods, including basic dyes, presents evidence which suggests that species differences in the structure of myofibrils occur, as shown by the differing relation of bands stained to birefringence in different animal types when using the same staining technique. This observation raises the question as to how much of the physiological and biochemical data obtained on other forms is directly applicable to mammalian, and in particular to human, muscle.

Dempsey, Wislocki and Singer ${ }^{3}$ (who worked with monkeys, rats and a newt, Triturus viridescens) claimed that basic dyes stained the anisotropic (or doubly refractile) segments, and that acidic dyes stained the isotropic (singly refractile) segments. Neither of these statements appears to be of general application. I have previously illustrated ${ }^{4}$ the appearances of human muscle as stained by the acidic dye fast green F.C.F. On that occasion, relying on the assumption of uniformity and the results of Dempsey, Wislocki and Singer, I misinterpreted the significance of the bands stained by this dye. In man, and other animals so far examined, it is not the isotropic but the anisotropic segments which are stained by acidic dyes.

These observations indicate that in considering the structure of myofibrils more attention should be paid to the possibility of species difference.

\section{Irvine J. Hunter*}

Department of Morbid Anatomy,

The Hospital for Sick Children, Great Ormond Street,

London, W.C.1. Aug. 13.

* Research Feliow in Histochemistry.

Carleton H. M. and Short, R. H. D. (edits.), "Schafer's Essentials of Hi stulugy", 16 th ed., 143 (Longmans, London, 1954).

"Meigs, E. B., in "Special Cytology", E. V. Cowdry (edit.), 2nd ed., 2, 1091 (Hoeber, New York, 1932).

${ }^{3}$ Dempsey, E. W., Wislocki, G. B., and Singer, M., Anat. Rec., 96, 221 $(1946)$.

${ }^{4}$ Hunter, I. J., Proc. Roy. Soc. Med., 51, 343 (1958).

\section{Effect of Chloramphenicol on a Fungus}

DURING an investigation of the formation of extracellular nitrogen compounds by fungi ${ }^{1}$, the effect of chloramphenicol on this process was tested, since in bacteria chloramphenicol is known to interfere with protein synthesis ${ }^{2}{ }^{3}$ and with the synthesis of enzymes concerned with amino-acid metabolism ${ }^{4}$, although it has no effect on the growth of many fungi ${ }^{5}$.

A strain of Scopulariopsis brevicaulis (Sacc.) Bainier was grown in surface culture in a synthetic glucose-nitrate liquid medium as used previously ${ }^{1}$, to which a trace metal supplement containing iron, copper, zinc, manganese and molybdenum was added. Chloramphenicol was added to some of the flasks at a final concentration of 20 or $50 \mu \mathrm{gm}$. $/ \mathrm{ml}$. In the control flasks growth of the fungus was rapid, all the nitrate was assimilated in 6-7 days, and the mean dry weight of mycelium after 20 days was $180 \mathrm{mgm}$. per flask. The extracellular nitrogen formed represented 16-20 per cent of the nitrogen supplied. In the presence of chloramphenicol, however, growth of fungus was very slow and nitrate did not finally disappear from the medium until 20-40 days after inoculation, when the dry weight of mycelium was 